\title{
A Compositional Semantics for Focusing Subjuncts
}

\author{
Daniel Lyons* \\ $\mathrm{MCC}$ \\ 3500 West Balcones Center Drive \\ Austin, TX 78759, USA \\ lyons@mcc.com
}

\author{
Graeme Hirst \\ Department of Computer Science \\ University of Toronto \\ Toronto, Canada M5S 1A4 \\ gh@ai.toronto.edu
}

\begin{abstract}
A compositional semantics for focusing subjuncts-words such as only, even, and also-is developed from Rooth's theory of association with focus. By adapting the theory so that it can be expressed in terms of a frame-based semantic formalism, a semantics that is more computationally practical is arrived at. This semantics captures pragmatic subtleties by incorporating a two-part representation, and recognizes the contribution of intonation to meaning.
\end{abstract}

\section{Introduction}

Focusing subjuncts such as only, even, and also are a subclass of the sentence-element class of adverbials (Quirk et al., 1985). They draw attention to a part of a sentence-the focus of the focusing subjunct-which often represents 'new' information. Focusing subjuncts are usually realized by adverbs, but occasionally by prepositional phrases. Focusing subjuncts emphasize, approximate, or restrict their foci. They modify the force or truth value of a sentence, especially with respect to its applicability to the focused item (Quirk et al., 1985, §8.116).

\subsection{The problem with focusing subjuncts}

There are several reasons why developing any semantics for focusing subjuncts is a difficult task.

First, focusing subjuncts are 'syntactically promiscuous'. They can adjoin to any maximal projection. They can occur at almost any position in a sentence.

Second, focusing subjuncts are also 'semantically promiscuous'. They may focus (draw attention to) almost any constituent. They can precede or follow the item that they focus, and need not be adjacent to this item. The focus need only be contained somewhere within the syntactic sister of the focusing subjunct. Because of this behavior, it is difficult to determine the intended syntactic argument (adjunct) and focus of a focusing subjunct. Sentences

"The work described in this paper was done at the University of Toronto. such as those in (1) can be ambiguous, even when uttered aloud with intonational effects. ${ }^{1}$

1. John could also 〈SEE $\rangle$ his wife from the doorway (as well as being able to talk to her).

2. John could also see (his wiFE) from the doorway (as well as her brother).

3. John could also see his wife from the Doorway) (as well as from further inside the room).

4. John could also (see his wife from the DOoRway) (as well as being able to do other things).

Third, the location of intonational stress has an important effect on the meaning of a sentence containing a focusing subjunct. Sentences may be partly disambiguated by intonational stress: interpretations in which stress falls outside the intended focus of the focusing subjunct are impossible. For example, the sentence

(2) *John could also see (his wife) from the DOoRway.

is impossible on the indicated reading, since stress on door cannot confer focus on his wife. On the other hand, stress does not help to disambiguate between readings such as (1.3) and (1.4).

Fourth, focusing subjuncts don't fit into the slotfiller semantics that seem adequate for handling many other sentence elements (see Section 1.3). At best, their semantic effect is to transform the semantic representation of the constituent they modify in some predictable compositional way (Hirst, 1987, p. 72).

Finally, focusing subjuncts carry pragmatic "baggage". The meaning of a focusing subjunct includes distinct asserted and non-asserted parts (Horn, 1969), (Karttunen and Peters, 1979). For example,

\footnotetext{
${ }^{1}$ In the example sentences in this paper, small capitals denote intonational stress. Angle brackets () enclose the focus of a focusing subjunct and square brackets [ ] set off the constituent to which the focusing subjunct adjoins. Unacceptable sentences are preceded by an asterisk.
} 
(3) asserts (4.1) but only presupposes (4.2) (Horn, 1969):

(3) Only Muriel voted for Hubert.

(4) 1. No one other than Muriel voted for Hubert.

2. Muriel voted for Hubert.

Analogously, (5) asserts (6.1) and presupposes (6.2) (Karttunen and Peters, 1979):

(5) Even Bill likes Mary.

(6) 1. Bill likes Mary.

2. Other people besides Bill like Mary; and of the people under consideration, Bill is the least likely to like Mary.

The precise status of such pragmatic inferences is controversial. We take no stand here on this issue, or on the definition of "presupposition". We will simply say that, for example, (4.1) is due to the asserted meaning of only, and that (4.2) is produced by the non-asserted meaning of only.

\subsection{Requirements of a semantics for focusing subjuncts}

We desire a semantics for focusing subjuncts that is compositional (see Section 1.3), computationally practical, and amenable to a conventional, structured, near-first-order knowledge representation such as frames. It must cope with the semantic and syntactic problems of focusing subjuncts by being cross-categorial, being sensitive to intonation, and by distinguishing asserted and nonasserted meaning. By cross-categorial semantics we mean one that can cope with syntactic variability in the arguments of focusing subjuncts.

We will demonstrate the following:

- Intonation has an effect on meaning. A focus feature is useful to mediate between intonational information and meaning.

- It is desirable to capture meaning in a multipart semantic representation.

- An extended frame-based semantic representation can be used in place of higher-order logics to capture the meaning of focusing subjuncts.

\subsection{Syntactic and semantic frameworks}

In this paper, we will use a compositional, framebased approach to semantics. Focusing subjuncts have been thought difficult to fit into a compositional semantics because they change the meaning of their matrix sentences in ways that are not straightforward.

A compositional semantics is characterized by the following properties:

- Each word and well-formed syntactic phrase is represented by a distinct semantic object.
- The semantic representation of a syntactic phrase is a systematic function of the representation of its constituent words and/or phrases.

In a compositional semantics, the syntax drives the semantics. To each syntactic phrase construction rule there corresponds a semantic rule that specifies how the semantic objects of the constituents are (systematically) combined or composed to obtain a semantic object for the phrase. Proponents of com positional semantics argue that natural language itself is for the most part compositional. In addition, using a composition semantics in semantic interpretation has numerous computational advantages.

The particular incarnation of a compositional semantics that serves as the semantic framework for this work is the frame-based semantic representation of Hirst's Absity system (Hirst, 1987, 1988). Absity's underlying representation of the world is a knowledge base consisting of frames. A frame is a collection of stereotypical knowledge about some topic or concept (Hirst, 1987, p. 12). A frame is usually stored as a named structure having associated with it a set of slots or roles that may be assigned values or fillers. Absity's semantic objects belong to the types in a frame representation language called Frail (Charniak, 1981). Absity uses the following types of semantic object:

- a frame name

- a slot name

- a frame determiner

- a slot-filler pair

- a frame description (i.e. a frame with zero or more slot-filler pairs)

- either an instance or frame statement (atom or frame determiner with frame description)

A frame determiner is a function that retrieves frames or adds them to the knowledge base. A frame description describes a frame in the knowledge base. The filler of a slot is either an atom, or it is an instance, specified by a frame statement, of a frame in the knowledge base. In order to capture the meaning of sentences containing focusing subjuncts, we will augment Absity's frame-representation language with two new semantic objects, to be described in Section 3.3.

The notation Hirst uses for frames is illustrated in Figure 1, which is a frame statement translation of the sentence

(7) Ross washed the dog with a new shampoo.

The semantics we will outline does not depend on any particular syntactic framework or theory. However, we choose to use Generalized Phrase Structure Grammar (GPSG) (Gazdar et al., 1985), because this formalism uses a compositional semantics that 


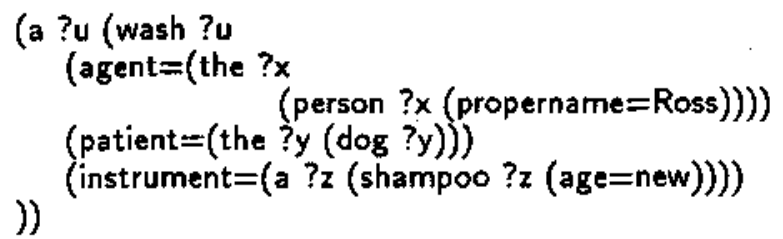

Figure 1: An Absity frame statement

resembles Montague grammar (Montague, 1973). A central notion of GPSG that we will make use of is that of the features of a syntactic phrase. A feature is a piece of linguistic information, such as tense, number, and bar level; it may be atom-valued or categoryvalued.

\subsection{Previous research}

The groundwork for the analysis of focusing subjuncts was laid by Horn (1969). Horn describes only (when modifying an NP) as a predicate taking two arguments, "the term $[x]$ within its scope" and "some proposition $[P x]$ containing that term" (Horn, 1969, p. 99). The meaning of the predicate is then to presuppose that the proposition $P$ is true of $x$, and to assert that $x$ is the unique term of which $P$ is true: $\neg(\exists y)(y \neq x \& P y)$. Even takes the same arguments. It is said to presuppose $(\exists y)(y \neq x \& P y)$ and to assert $P_{x}$. Horn requires a different formulation of the meaning of only when it modifies a VP. Since his formulation is flawed, we do not show it here.

Jackendoff's (1972, p. 242) analysis of even and only employs a semantic marker $F$ that is assumed to be present in surface structure and associated with a node containing stress. He calls the semantic material associated with constituents marked by $F$ the focus of a sentence. He proposes a rule that states that even and "related words" are associated with focus by having the focus in their range. Differences between the ranges of various focusing adverbs account for their different distributions (Jackendoff, 1972, pp. 249-250). For example:

Range of even: If even is directly dominated by a node $X$, then $X$ and all nodes dominated by $X$ are in its range.

Range of only: If only is directly dominated by a node $X$, then $X$ and all nodes that are both dominated by $X$ and to the right of only are in its range.

That is, only cannot precede its focus (nor can just, which has the same range), but even can:

1. * (JoHN $\rangle$ only gave Mary a birthday present (no one else did).

2. 〈JoHN〉 even gave Mary a birthday present (and so did everyone else, but
John was the person least expected to).

We will employ several aspects of Rooth's (1985) domain selection theory. A key feature of the theory is that only takes the VP adjacent to it in S-structure as its argument (an extension of the theory allows only to take arguments other than VPs). Rooth describes technical reasons for this arrangement (1985, p. 45). Among these is the fact that focusing subjuncts can draw attention to two (or more) items that, syntactically, do not together constitute a well-formed phrase:

(9) John only introduced $\langle$ BILL $\rangle$ to $\langle$ SUE $\rangle$.

The prevailing linguistic theories allow a node (such as a focusing subjunct) only one argument in the syntactic or logical (function-argument) structures of a sentence.

According to Rooth, the asserted meaning of

(10) John only [ $v P$ introduced BiLL to Sue].

is "if John has a property of the form 'introduce $y$ to Sue' then it is the property 'introduce Bill to Sue'" (Rooth, 1985, p. 44, p. 59). Rooth's theory would produce the same translation, shown in (11.2), for both sentence (10) and sentence (11.1).

1. John only introduced Bill to SuE:

2. $\forall P[[P(j o h n) \& P \in C]$

$$
\left.\rightarrow P={ }^{\wedge} \text { introduce' }(\text { bill, sue })\right]
$$

$P$ ranges over propositions, so (11.2) is a quantification over propositions. $C$ is bound ${ }^{2}$ to the p-set of the VP of whichever sentence's meaning (11.2) is intended to capture. This p-set is "a set of properties, which we think of as the set of relevant properties" (Rooth, 1985, p. 43).

Different truth conditions for the two sentences (10) and (11.1) obtain because their VPs have different p-sets: the computation of p-sets is sensitive to intonational stress (actually to focus, which is signalled by stress; see below). The desired value for $C$ in the translation of (10) is the set of propositions of the form "introduce $y$ to Sue", namely propositions satisfying (12.1). For the translation of (11.1), $C$ is the set of propositions of the form "introduce Bill to $y$ ", that is, those satisfying (12.2).

$$
\begin{aligned}
& \text { 1. } \lambda P \exists y\left[P={ }^{\wedge} \text { introduce }(y, s u e)\right] \\
& \text { 2. } \lambda P \exists y\left[P={ }^{\wedge} \text { introduce }(\text { bill }, y)\right]
\end{aligned}
$$

These result in the final translations (13.1) and (13.2) respectively for sentences (10) and (11.1):

$$
\begin{aligned}
& \text { 1. } \forall y[\text { introduce }(j o h n, y, \text { sue }) \rightarrow y=b i l l] \\
& \text { 2. } \forall y[\text { introduce }(j o h n, b i l l, y) \rightarrow y=s u e]
\end{aligned}
$$

\footnotetext{
${ }^{2}$ The mechanism of this binding relies on the translation being a formula of which (11.2) is a reasonable simplification; see (Rooth, 1985, p. 59).
} 
The formula (13.1) corresponds to the gloss of the meaning of (10) given above. (13.2) is to be interpreted as meaning: "if John has a property of the form 'introduce Bill to $y$ ' then it is the property 'introduce Bill to Sue'".

The p-set of a complete sentence is a set of "relevant propositions". Rooth defines it recursively, from the p-sets of its constituents (Rooth, 1985 , p. 14) (the "model" is a Montague-style formal model):

(14) Let a be a constituent with translation $a^{\prime}$. The p-set of $a$ is:

1. if $a$ bears the focus feature, the set of objects in the model matching $a^{\prime}$ in type;

2. if $a$ is a non-focused non-complex phrase, the unit set $\left\{a^{\prime}\right\}$;

3 . if $a$ is a non-focused complex phrase, the set of objects that can be obtained by picking one element from each of the p-sets corresponding to the component phrases of $a$, and applying the semantic rule for $a$ to this sequence of elements.

In other words, the p-set of a sentence consists essentially of all propositions that are "like" the proposition that it asserts, except that the focused constituent in the proposition is replaced by a variable. ${ }^{3}$

We will adopt Rooth's definition of the meaning of only: A sentence containing only that (without only) has logical form $a$ :

1. asserts that any "contextually relevant" proposition $P$ whose extension is true is the proposition $a$;

2. has $a$ as part of its non-asserted meaning. (Rooth, 1985, p. 120).

Our analogous definition of even is this: A sentence containing even that (without even) has logical form a:

1. asserts $a$;

2. conveys the non-asserted inference that there are other "contextually relevant" propositions, besides $a$, that are true.

\section{Devices used to solve the problems}

Our semantics (which is described in more detail by Lyons (1989)) employs devices described in the following sections.

\subsection{The focus feature}

Following Jackendoff, we propose that focus is a binary feature, similar to (say) gender and number,

${ }^{3}$ The notion that the meaning of only and even can be defined in terms of a base form (such as "John introduced $y$ to Sue") was also noted by Karttunen and Peters (1979) and McCord (1982). that is either present or absent on every constituent at surface structure. ${ }^{4}$ Focus is initially instantiated onto the leaves of the tree that represent intonationally stressed words. The only realization of the focus feature that we accommodate is intonational accent; however, our theory can easily be extended to allow for other overt realizations of focus, including other intonational effects (e.g. (Hirschberg and Pierrehumbert, 1986)). Focus is optionally and nondeterministically percolated up the syntax tree, to any node from its rightmost daughter (rightmost because stress manifests itself only at the end of the focused constituent (Anderson, 1972)). The nondeterminism of the percolation of focus is responsible for ambiguity in the interpretation of sentences with focusing subjuncts. How far the focus feature percolates up determines how wide a focus is attributed to the focusing subjunct:

1. John also read the book (from the LIBRARY) (as well as the one from the store).

2. John also read (the book from the LIBRARY) (as well as the newspaper).

3. John also (read the book from the LIBRARY) (as well as completing his assignment).

The ambiguous interpretations of a sentence with a focusing subjunct belong to an ordered set in which each reading has a wider focus for the focusing subjunct than the previous one.

\subsection{Relevant propositions}

Our semantics employs a computational analogue of Rooth's p-sets for a frame representation. Our pset for a constituent is computed compositionally, along with the semantic representation, in tandem with the application of the syntactic rule used to build the constituent. The p-set turns out to be an object in the frame representation that is like the semantic assertion derived for the constituent, but lacking restrictive information associated with any focused components.

\subsection{Two-part semantics}

In addition to p-sets, two semantic expressions are computed for each constituent during the interpretation of a sentence. One expression represents asserted meaning, and the other, non-asserted meaning.

\footnotetext{
This feature is what Jackendoff calls the $F$ marker, but is different from what he calls "focus". Note that we use the term focus of a focusing subjunct to stand for a distinct concept: the item to which a focusing subjunct draws attention to, or focuses. This is the semantic material that corresponds to a stressed word or to a constituent containing one.
} 


\subsection{Linguistic features}

Focus is marked as a binary feature on all syntactic constituents. The semantic rules use this information when constructing semantic expressions for constituents. Because the focus feature need not percolate all the way up to the level of the constituent that is adjacent to the focusing subjunct in the syntax tree, we have found it useful to employ a second feature, focus-in, that indicates whether or not any sub-phrase is focused. The restriction that a focusing subjunct adjoins only to a phrase containing focus is implemented by requiring the adjunct phrase to be (focus-in + ).

Range (see Section 1.4) is implemented as two binary features, range-right and range-left, that indicate whether or not a given focusing subjunct can adjoin to phrases to its right and left, respectively. (Some words, like even, have both features.)

\subsection{Sentential operators}

Rooth applies his even and only operators to the logical form of the constituent that is the syntactic sister of the focusing subjunct. So, for example, in the VP (18.1), only transforms the expression wash'(dog), which is the translation of the VP argument of only, into the $\lambda$-expression (18.2).

$$
\begin{aligned}
& \text { 1. only [VP washed the }\langle\mathrm{DoG}\rangle] \\
& \text { 2. } \lambda_{x} \forall P\left[{ }^{\vee} P \& P \in C\right] \\
& \left.\qquad P={ }^{\wedge}{ }^{\wedge} \operatorname{wash}^{\prime}(x, \operatorname{dog})\right]
\end{aligned}
$$

For each focusing subjunct, Rooth must define a separate transformation for each different semantic type of phrase that it may take as an argument. He defines a basic sentential operator for each focusing subjunct, and then derives the other operators from these (Rooth, 1985, pp. 120-121).

Our approach is to instead define a single operator for each focusing subjunct, essentially Rooth's basic sentential operator. This operator takes the semantic representation of a sentence as an argument and produces another semantic representation of sentential type. When sentential objects are not available, as in the interpretation of [ $V P$ only VP], we delay the application of the operator until such a point as fully developed propositions, the semantic objects of sentences, are available. To do this, the grammar rules "percolate" focusing subjunct operators up the syntax tree to the $S$ node. Our grammar employs the feature $f s$ to carry this latent operator. When the interpretation of a sentence is otherwise completed, a final step is to apply any latent operators, producing expressions for the sentence's asserted and nonasserted meanings from expressions for its assertion and its p-set.

Several pieces of evidence motivate this approach:

- As Rooth observed, in order to define a family of cross-categorial operators for (say) only, a basic operator must be defined that operates on an expression of sentential type. The semantics of focusing subjuncts actually seems to take place at the sentence level.

- Focusing subjuncts normally occur at most once per sentence. Even granting the acceptability of sentences containing several focusing subjuncts, such sentences are clearly semantically complicated.

The principal advantage of our approach is that it constructs essentially the same final translation of a sentence as Rooth's, but avoids using the $\lambda$ operator during the derivation of a semantic representation that does not itself contain a $\lambda$-operator. This is desirable, as $\lambda$-expressions would make the frame representation language less tractable.

\section{Details of the semantics}

\subsection{Semantic features}

Three semantic objects are computed for and attached to each syntactic constituent, in parallel with the syntactic processing. The objects are of the types defined in an Absity-like frame representation. They are attached to a node as values of the following features (an approach motivated by Shieber (1986)):

Assert: The asserted meaning of the constituent, its contribution to the sentence's asserted meaning. The value is computed the same way that a Montague-style grammar would construct a constituent's logical form from those of its daughters. Figure 2 shows examples of the rules to compute this value.

Presupp: The constituent's contribution to the sentence's non-asserted meaning. For all rules but sentence rules, the presupp feature on the parent node is undefined. In order not to commit ourselves to the status of the non-asserted meanings of focusing subjuncts, we reserve this feature for the non-asserted meanings introduced by focusing subjunct operators (see below).

P-set: A prototype of the semantic objects in the node's p-set. All objects that match this object are in the node's p-set. The algorithm for computing p-sets distinguishes between two cases:

Case 1: If the parent node $X$ (being constructed) is (focus + , its $p$-set is a variable of the same type as the assert object.

Case 2: Otherwise, the p-set of $X$ is constructed from the p-set values of the constituent phrases in a manner exactly paralleling the construction of the assert feature. 


\begin{tabular}{|c|c|c|}
\hline & Syntax rule & Semantic rule \\
\hline$S \rightarrow$ & $\begin{array}{l}\mathrm{XP}[(\text { assert (agent }=\alpha)\rangle] \\
\operatorname{VP}[(\text { assert (frame } \beta \text { sf-pairs }))]\end{array}$ & $\mathrm{S}=\mathrm{S}[$ (assert (frame $\beta$ (agent $=\alpha$ ) sf-pairs)) $]$ \\
\hline $\mathrm{VP} \rightarrow$ & $\begin{array}{l}\mathrm{V}[2 \text { (assert }(\text { frame } ? \alpha)\rangle] \\
\mathrm{NP}[\mathrm{obj} \text { (assert }(\operatorname{slot} \beta=\beta)\rangle]\end{array}$ & $\mathrm{VP}=\mathrm{V}[\langle$ assert $($ frame $? \alpha(\operatorname{slot} \beta=\beta))\rangle]$ \\
\hline $\mathbf{P P} \rightarrow$ & $\begin{array}{l}\mathrm{P}[38 \text { (assert slot } \alpha\rangle] \\
\mathrm{NP} \mid \text { (assert } \beta)]\end{array}$ & $\mathrm{PP}=\mathrm{PP}[\langle$ assert $(\operatorname{slot} \alpha=\beta)\rangle]$ \\
\hline
\end{tabular}

Figure 2: Examples of semantic rules for the assert feature

\subsection{Application of the focusing subjunct operators}

There is a syntactic rule whose sole purpose is to support of the application of a sentential operator:

$$
\mathrm{S} \rightarrow \mathrm{H}[\langle\mathrm{fs} \alpha\rangle]
$$

$\mathrm{S}[\mathrm{fs} \alpha]$ is specified as a non-initial category in the grammar, if $\alpha \neq$ " $-"$. Therefore, the rule (19) must apply in the derivation of any well-formed sentence containing a focusing subjunct. The corresponding semantic rule (20) applies a focusing subjunct operator to the semantic representation of the sentence.

$$
\begin{aligned}
& \text { 1. Input: } \\
& \mathrm{S}[(\text { assert } \alpha\rangle,\langle\text { p-set } \beta\rangle,\langle\mathrm{fs} \gamma\rangle]
\end{aligned}
$$

2. Output:

$$
\begin{aligned}
& \text { - If } \gamma=\text { "-" then } \\
& \text { - }[\langle\text { assert } \alpha\rangle,\langle\text { p-set } \beta\rangle] \\
& \text { - else } \\
& \quad \mathrm{S}\left[\begin{array}{l}
\text { assert op } \left.1_{\gamma}(\alpha, \beta)\right\rangle, \\
\text { presupp op2 } \left.2_{\gamma}(\alpha, \beta)\right\rangle,
\end{array}\right. \\
& \quad \text { p-set } \beta\rangle]
\end{aligned}
$$

where op $1_{\gamma}$ and op2 $\gamma$ are the sentential operators for the focusing subjunct $\gamma$ (see below).

\subsection{The sentential operators}

The sentential operators for only and even are given below. (The one for too is the same as that for even, and those for the other focusing subjuncts are similar.)

$$
\begin{aligned}
& \text { 1. op } 1_{\text {oniy }}(A, P)=\text { if } P \text { then } A \\
& \text { 2. } \mathrm{op}_{\text {only }}(A, P)=A \\
& \text { 3. } o p 1_{\text {even }}(A, P)=A \\
& \text { 4. op2 even ( (the ? } x \text { frame-descrA), } \\
& \text { (the ?y frame-descr } P \text { ) ) } \\
& =\text { (anew } ? y \neq ? x \text { (frame-descrP)) }
\end{aligned}
$$

The form if $P$ then $A$ is a directive to the underlying knowledge base to insert the rule that any frame matching $P$ is just the frame $A$, that is, $A$ is the unique frame matching $P$. This directive is a frame implication. It is similar in character to a frame determiner (Hirst, 1987), in that it is a function that manipulates the underlying knowledge base.

The form (anew ? $y \neq ? x$ frame-descrP) is also a new type of entity in the semantics. We treat it as a frame determiner. It is a directive to the knowledge base to retrieve or create a frame instance, ? $y$, that matches frame-descr $\mathrm{P}$ but is not the frame instance identified by the variable ? $x$. As with the frame determiner (the ?x), such a frame instance ? $y$ should be inserted if not already present in the knowledge base.

For example, the sentence (22.1) yields the expression (22.2) as its assertion and (22.3) as its non-asserted meaning (other readings are possible as well).

1. Ross only washed the Dog.

2. if (wash ? $x$ (agent=Ross)) then

(wash ? $x$ (agent=Ross) (patient=dog)))

3. (the ?x (wash ? $x$ (agent=Ross) (patient $=$ dog)))

The frame instance (22.3) captures the semantic content of the sentence "Ross washed the dog". The frame implication (22.3) is to be interpreted as the rule that any wash frame in the knowledge base having Ross as its agent must in addition have dog as its patient.

A second example: sentence (23.1) yields assertion (23.2) and non-asserted meaning (23.3).

(23) 1. Ross washed even the Doc.

2. (the ? $x$ (wash ? $x$ (agent=Ross) (patient=dog)))

3. (anew $? y \neq ? x$ (wash ? $y$ (agent=Ross)))

The expression (23.3) affirms the existence of a wash instance ? $y$ having agent Ross but that is a distinct washing from ? $x$ in (23.2), which has dog as its patient.

\section{The implementation}

IDEo (Interpreter Designed for Even and Only) is a limited semantic interpreter that incorporates the 
semantics for even and only described in Section 3. The implementation is in Edinburgh C-Prolog, running under UNIX on a Sun-4 computer. Because the authors did not have access to a working version of Frail (see Section 1.3), IDEo runs on top of a toy knowledge base, also implemented in C-Prolog, whose retrieval language is (unfortunately) a syntactic variant of Absity's.

A sample session with IDEo is follows below. In this trace, output generated by the program or typed by the user is shown in typewriter font. As the example shows, IDEO accepts a sentence typed at the keyboard and outputs four different things. Firstly, it echoes the sentence in its internal Prolog format. Secondly, the GPSG category obtained for the sentence, which incorporates a parse tree for the sentence, is displayed. For the sake of readability, IDEo prints a simplified version of the category. Then, it prints separately the semantic representation of the sentence, which consists of several of the featurevalue pairs of the sentence category. Lastly, it displays a message about the success or failure of each knowledge base function call resulting from processing the semantic representation of the sentence. In a question-and-answer system, these messages would be replaced by generated English answers.

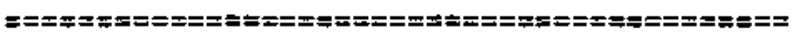
Please enter a sentence, followed by a carriage return, after the "l:" prompt.

I: Ross only washed the :dog.

The colon preceding the word dog tells IDEo that the word is intonationally stressed.

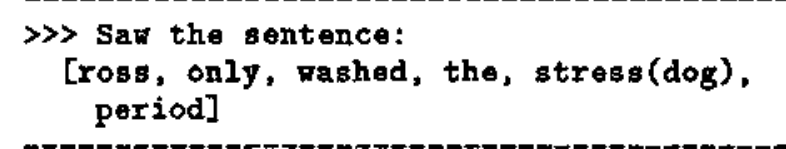

>> The category for this sentence is:

[Omitted due to space limitations.]

The significant piece of information in the GPSG category is that the noun phrase [NP the stress $(\operatorname{dog})]$ is (focus +$\rangle$, but the verb phrase that contains it is not.

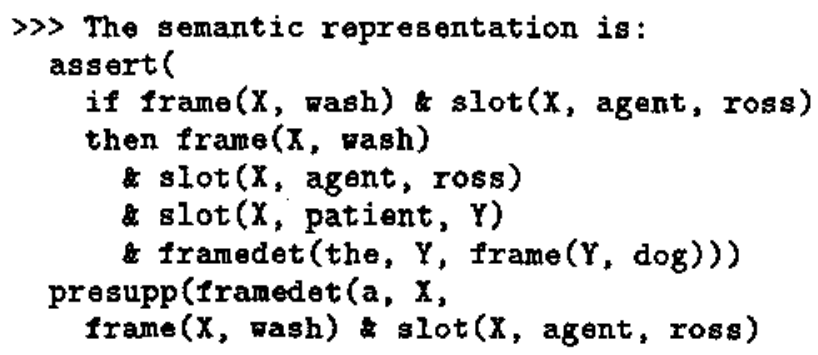

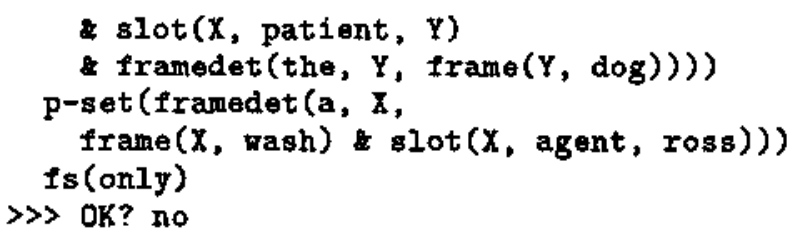

This semantic representation corresponds to the reading in which the speaker means to assert that Ross washed nothing else but the dog. The user replies no to the prompt $0 K$ ? to indicate that this is not the reading he intends.

\> The category for this sentence is:

[Omitted due to space limitations.]

This time, a parse is returned in which the feature (focus + ) percolates up the parse tree to the entire verb phrase [NP washed the stress (dog)].

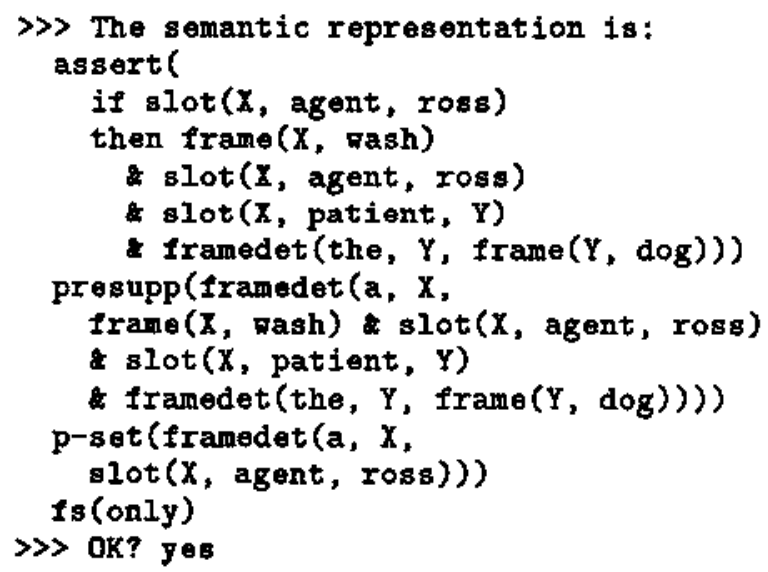

The user approves this semantic representation, which corresponds to the reading in which the speaker asserts that Ross did nothing but wash the dog.

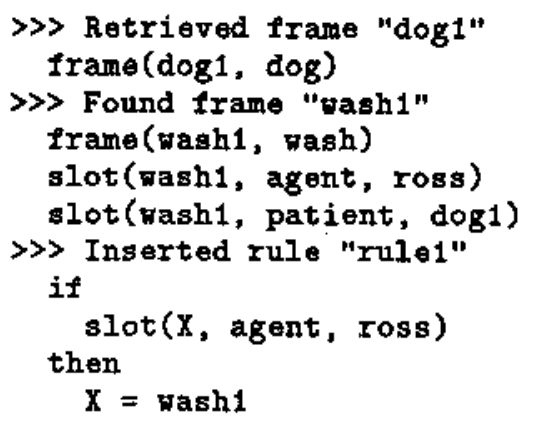

The knowledge base now is constrained by the rule rule1. This says that if a frame $x$ satisfies the frame description slot ( $x$, agent, ross) (i.e. its agent is ross), then it must be the frame wash1 (a wash frame whose patient slot is filled by $\operatorname{dog} 1$ ). 


\section{Conclusions}

This paper summarizes work, details of which can be found in (Lyons, 1989). The work represents a step towards better understanding of focusing subjuncts and of compositional semantics in general. The semantics we have proposed allows focusing subjuncts to be covered by a frame-based approach to semantic interpretation, by virtue of its being compositional, computationally practical, able to differentiate between asserted and non-asserted meaning, sensitive to intonation, and cross-categorial. We have found that:

- Focus and stress information can be used to advantage in a semantic interpreter.

- The hypothesis that focus may be optionally percolated to a parent node from a daughter explains the scope ambiguities observed in the interpretation of focusing subjuncts.

- Rooth's method of obtaining the translation of a focusing subjunct by using p-sets to select "domains of quantification" can be adapted to translating a sentence into a frame representation.

- Treating focusing subjuncts as operators on sentential semantic forms makes this translation possible.

- Semantically, focusing subjuncts are not just passive objects for composition. We have shown extensions to standard frame representations that are required for the translation of focusing subjuncts.

\section{Acknowledgements}

Both authors acknowledge the support of the Natural Sciences and Engineering Research Council of Canada. We are also grateful to Diane Horton, Brendan Gillon, Barb Brunson, and Mark Ryan for discussions, comments on earlier drafts, and general encouragement.

\section{References}

Anderson, Stephen R. (1972). How to get even. Language, 48:893-906.

Charniak, Eugene (1981). A common representation for problem-solving and language-comprehension information. Artificial Intelligence, 16(3):225-255. Also published as technical report CS-59, Department of Computer Science, Brown University, July 1980.

Gazdar, Gerald, Klein, Ewan, Pullum, Geoffrey K., and Sag, Ivan (1985). Generalized Phrase Structure Grammar. Harvard University Press.

Hirschberg, Julia and Pierrehumbert, Janet (1986). The intonational structuring of discourse. In $24^{\text {th }} \mathrm{An}$ nual Meeting of the Association for Computational
Linguistics, Proceedings of the Conference. pages 136-143.

Hirst, Graeme (1987). Semantic Interpretation and the Resolution of Ambiguity. Cambridge University Press.

Hirst, Graeme (1988). Semantic interpretation and ambiguity. Artificial Intelligence, 34(2):131-177.

Horn, Laurence R. (1969). A presuppositional analysis of only and even. In Binnick, Robert I., Davison, Alice, Green, Georgia, and Morgan, Jerry, editors, Papers from the Fifth Regional Meeting of the Chicago Linguistics Society. Chicago Linguistic Society, pages 98-107.

Jackendoff, Ray S. (1972). Semantic Interpretation in Generative Grammar. The MIT Press.

Karttunen, Lauri and Peters, Stanley (1979). Conventional implicature. In $\mathrm{Oh}$, Choon-Kyu and Dinneen, David A., editors, Presupposition, volume 11 of Syntax and Semantics. Academic Press, pages 156.

Lyons, Dan (1989). A computational semantics for focusing subjuncts. Master's thesis, Department of Computer Science, University of Toronto. Also published as technical report CSRI-234.

McCord, Michael C. (1982). Using slots and modifiers in logic grammars for natural language. Artificial Intelligence, 18:327-367.

Montague, Richard (1973). The proper treatment of quantification in ordinary English. In Hintikka, Kaarlo Jaakko Juhani, Moravcsik, Julius Matthew Emil, and Suppes, Patrick Colonel, editors, Approaches to Natural Language: Proceedings of the 1970 Stanford workshop on grammar and semantics. D. Reidel, pages 221-242. Also in Thomason, Richmond Hunt (ed.), Formal philosophy: Selected papers of Richard Montague. Yale University Press (1974): 247-270.

Quirk, Randolph, Greenbaum, Sidney, Leech, Geoffrey, and Svartvik, Jan (1985). A Comprehensive Grammar of the English Language. Longman.

Rooth, Mats Edward (1985). Association with Focus, PhD thesis, Department of Linguistics, University of Massachusets.

Shieber, Stuart M. (1986). An Introduction to Unification-Based Approaches to Grammar. Center for the Study of Language and Information. 